\title{
Introduction to Digital Design: A Paradigm-based Approach
}

\author{
Miodrag Potkonjak \\ Computer Science Department \\ Univ. of California, Los Angeles \\ miodrag@cs.ucla.edu
}

\author{
Jennifer Wong \\ Computer Science Department \\ SUNY Stony Brook University \\ jwong@cs.sunysb.edu
}

\begin{abstract}
Introduction to digital design is a standard undergraduate course in computer science, and electrical and computer engineering curriculums at many universities. While there are a wide variety of teaching approaches which emphasize different topics and aspects of digital design, there are numerous strong conceptual and education similarities. We present three strategic objectives in this article. The first is to summarize our extensive experiences teaching, searching, and developing conceptually new and highly effective ways to prepare students in designing digital systems. The second objective is to present a paradigm-based teaching approach for designing large scale and detailed digital designs. The approach emphasizes structured heuristic thinking and the application of reusable synthesis rules and templates. Finally, to make the educational material intuitive and interactive, we have developed a set of games which leverage on design knowledge and skills for effective play.
\end{abstract}

\section{Motivation}

"Introduction to Digital Design" is a standard prerequisite design and architecture course in essentially all computer engineering, and many computer science and electrical engineering curriculums. A large variety of these courses place emphasis on the use of mathematical and formal rigor, the use of CAD tools, a designer's viewpoint, industrial practice, and clarity of basic techniques $[3,2,4,6,7,8,9]$. Although the rapid progress of silicon technology and the scale of integration have been continuously changing, the presented course materials, such as dominant design abstractions, models, objectives and constraints, have undergone little change.

We place emphasis on four technical goals in our teaching. The first is to ensure that the students develop strong and practical skills to design. Secondly, the goal is to prepare them for consequent architecture, embedded systems, and system software courses. We induce them to think and act at the system level. And finally, the goal is to intro- duce students to sound algorithmic, probabilistic, and statistical techniques for the analysis of digital designs. We believe that the presented new paradigm-based approach, which follows, facilitates all four of these goals and provides a stronger foundation for current and developing technologies. Note, that paradigm-based teaching is often used in mathematics and computer science, however if is rarely used in design courses $[1,5]$.

\section{Paradigms}

The most common organizational approach for an introductory course in digital design is to cover the following material, in order: combinatorial gate-level logic synthesis and analysis, sequential gate-level logic synthesis and analysis, combinatorial modules, sequential modules, and finally system design. In addition, number systems are almost always covered. While we still follow this organization, we present each topic from the perspective of each of the following fundamental design paradigms: (i) parallelism; (ii) pipelining; (iii) separation of concerns; (iv) divide and conquer; (v) transform and conquer; and (vi) equivalent software. For example, in the current engineering curriculum, students are exposed to a variety of seemingly disparate components and models in different courses. Topics, such as communications, digital signal processing, architecture, application-specific integrated circuit design, and operating systems, are taught in a variety of settings preventing students from obtaining an integrated system view of the components in one framework. Another example is the teaching of network layers, the separation of control and data paths in microprocessors, and isolated compression and error correction steps in communication. All of these topics fall under a single design paradigm, separation of concerns. Sadly, their non-unified presentation prevents the students from grasping the underlying paradigm. To address this issue, we place an emphasis on the separation of concerns paradigm at various aspects of digital design. Such aspects include designing datapath and control logic, adder logic into carry-look-ahead components and the remainder standard, counters and controllers, and the design of the minimal required combinatorial logic. 


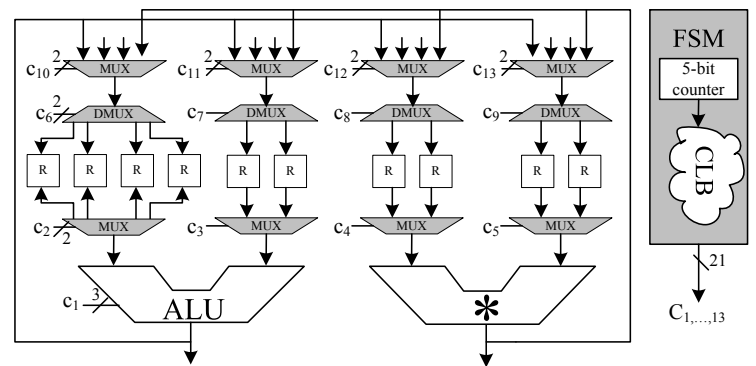

Figure 1. Illustration of an application specific architecture.

\section{Covered Material}

Through multiple series of teachings, we have experimented with covering different subsets of the standard digital design material. At the current time, we are settled on an inverse pyramid coverage of each topic. We cover the selected material in significant depth, at the expense of covering a smaller subset of topics. For example, at the integrated circuits level, we cover only the design of NAND and NOR gates. But at module and system design level, we cover both a set of application specific and a general purpose architectures. Figure 1 shows an illustrative example of one of our application specific architectures with two functional units, dedicated registers, and finite state machine (FSM).

\section{Games To Promote Design Techniques}

There is a persistent problem in engineering and scientific classes related to maintaining the students attention level and creating a lasting impetus for exploring the new concepts and acquiring skills through hands-on problem solving and design. Experience has shown that this problem can be addressed through two main notions. The first is motivating the topic through a brief discussion of design techniques in state-of-the-art gadgets, start-ups, and current directions of major semiconductor companies such as Intel, TI, NEC, and Toshiba. This discussion leads the students to see the underlying meaning of the material and very often stimulates the interests of the students. The second approach is our most revolutionary step. We create and maintain the interest of students through the development of digital design games. We have developed both video games, as well as chalkboard games, which are played by two or more players. For example, we developed a game where the goal of one player is to minimize the sum-of-products (SOP) and the goal of his opponent is to minimize productof-sums (POS) form in a Karnaugh map. The players either specify a minterm or a maxterm to be added interchangeably. Overall student response has been high. Interestingly, interpersonal games featuring the students, and in particular when of the players was the professor or the teaching assistant, were more popular than their video implementations.

\section{Assignments}

Plagiarism is widely recognized as one of dominant problems in education. In particular, the situation is difficult when a non-trivial percentage of the students overall score is based on assignments. In order to asses the magnitude of the problem, we minimally changed three homework problems that were given the previous year in the next course offering. More than three-fourths of the assignments contained the answers to the previous versions and several students even voiced their objections towards the length of the solutions. A very effective solution we found is to assign a large number of problems to the students. No solutions are turned-in, but a subset of the problems are selected for bi-weekly quizzes. We observed an increase in motivation for many students to learn all or a majority of the problems and the fundamental design techniques as well, rather than just completing the assignment.

\section{Evaluation and Conclusion}

We evaluated new approach to introductory digital design class using four metrics. The first metric was student evaluation scores. Consistently, the digital design scores were in the top 10\% of UCLA School of Engineering and Science in terms of the lecturer and perceived course benefits. Maybe more importantly, attendance was consistently close to complete. In ABET evaluations, one of the offering received the best evaluation of all UCLA Computer Science Department offerings. Finally, by tracking the students in corresponding consequent laboratory courses indicated that they often have superior performance. In the future, we plan to calibrate the performance of the paradigm-based digital design student with other methodologies by offering common exams between simultaneously course offerings.

We summarized a paradigm-based approach to teaching an introductory digital design course. The high evaluations and student participation reflect positively on a course instructed through underlying paradigm principles and interactive games.

\section{References}

[1] R. A. Baeza-Yates. Teaching algorithms. SIGACT News, 26(4):51-59, 1995.

[2] M. Ercegovac, J. Moreno, and T. Lang. Introduction to Digital Systems. John Wiley \& Sons, 1998.

[3] D. Gajski. Principles of digital design. Prentice-Hall, 1996.

[4] R. H. Katz. Contemporary Logic Design. Benjamin Cummings/Addison Wesley Publishing Company, 1993.

[5] A. Levitin. Introduction to the Design and Analysis of Algorithms. Addison-Wesley Longman Publishing Co., 2002.

[6] M. Mano. Digital Design. Prentice Hall PTR, 2001.

[7] F. Vahid. Digital Design. John Wiley \& Sons, 2006.

[8] J. F. Wakerly. Digital design: principles and practices. Prentice Hall, 2005.

[9] M. Zwolinski. Digital System Design with VHDL. AddisonWesley Longman Publishing Co., 2000. 